PROCEEDINGS OF THE

AMERICAN MATHEMATICAL SOCIETY

Volume 131, Number 4, Pages 1093-1102

S 0002-9939(02)06647-9

Article electronically published on September 5, 2002

\title{
REGULARITY OF LEX-SEGMENT IDEALS: SOME CLOSED FORMULAS AND APPLICATIONS
}

\author{
MARC CHARDIN AND GUILLERMO MORENO-SOCÍAS
}

(Communicated by Wolmer V. Vasconcelos)

\begin{abstract}
A closed formula for computing the regularity of the lex-segment ideal in terms of the Hilbert function is given. This regularity bounds the one of any ideal with the same Hilbert function. As a consequence, we give explicit expressions to bound the regularity of a projective scheme in terms of the coefficients of the Hilbert polynomial.

We also characterize, in terms of their coefficients, which polynomials are Hilbert polynomials of some projective scheme.

Finally, we provide some applications to estimates for the maximal degree of generators of Gröbner bases in terms of the degrees of defining equations.
\end{abstract}

\section{INTRODUCTION}

Macaulay proved the existence of a lex-segment ideal associated to a Hilbert function. The Castelnuovo-Mumford regularity of a lex-segment ideal depends only on the Hilbert function and bounds the regularity of any ideal with the same Hilbert function (in fact every graded Betti number is bounded by the corresponding one of the lex-segment ideal, as was proved by Bigatti and Hulett in characteristic zero and by Pardue in general).

Here we give a closed formula for computing the regularity of the lex-segment ideal in terms of the Hilbert function (Lemma 1.5 and Theorem 2.5 (i)). As a consequence, we are for example able to characterize, in terms of their coefficients, which polynomials are Hilbert polynomials of some projective scheme.

We also give explicit expressions to bound the regularity of a projective scheme in terms of its Hilbert polynomial (Corollary 2.6). This relies on our previous computations and a result of Gotzmann (Go) (Theorem 2.5 (ii), for which we provide an alternative argument).

In the last section we provide some applications to estimates for the maximal degree of generators of Gröbner bases in terms of the degrees of defining equations (Corollary [3.6). These bounds are huge, as they should be, as shown by MayrMeyer examples. They may be used to bound the regularity of the initial ideal of the associated primes of a complete intersection for any admissible order and any coordinates (Corollary [3.7). These bounds rely on an estimate of the coefficients of the Hilbert polynomial of a graded complete intersection, and on our closed formulas for the regularity of a lex-segment ideal in terms of the Hilbert

Received by the editors May 18, 2001 and, in revised form, December 4, 2001.

2000 Mathematics Subject Classification. Primary 13D02, 13D40, 13D45, 13 P10. 
polynomial. It should be noted that these bounds are very far from the expected ones in terms of Castelnuovo-Mumford regularity; this reflects the lack of results for general irreducible projective schemes.

\section{Macaulay Representation of polynomials}

Notation 1.1. (1) If $m, \mu$ are two positive integers, there is a unique set of integers $c_{\mu} \geq c_{\mu-1} \geq \cdots \geq c_{\mu-b+1} \geq 0$ with $b \leq \mu$ such that

$$
m=\left(\begin{array}{c}
c_{\mu}+\mu \\
\mu
\end{array}\right)+\left(\begin{array}{c}
c_{\mu-1}+\mu-1 \\
\mu-1
\end{array}\right)+\cdots+\left(\begin{array}{c}
c_{\mu-b+1}+\mu-b+1 \\
\mu-b+1
\end{array}\right) .
$$

We will write $m=\left\langle c_{\mu}, c_{\mu-1}, \ldots, c_{\mu-b+1}\right\rangle_{\mu}$. We will also use another abbreviated notation,

$$
m=\left[b_{c_{\mu}}, b_{c_{\mu}-1}, \ldots, b_{0}\right]_{\mu},
$$

where $b_{i}:=\#\left\{j \mid c_{j}=i\right\}$, and set $m^{\langle\mu\rangle}:=\left[b_{c_{\mu}}, b_{c_{\mu}-1}, \ldots, b_{0}\right]_{\mu+1}$. Notice that $b=b_{0}+\cdots+b_{c_{\mu}}$.

(2) If $P \in \mathbb{Q}[t]$ is a polynomial, we can write

$$
P(t)=a_{d}\left(\begin{array}{c}
t+d \\
d
\end{array}\right)+\cdots+a_{1}\left(\begin{array}{c}
t+1 \\
1
\end{array}\right)+a_{0}
$$

with $d=\operatorname{deg} P$ and we will set $P=\left(a_{d}, a_{d-1}, \ldots, a_{0}\right)$.

(3) For $j$ a non-negative integer and $w$ in a commutative ring $A$, we let $S$ be the multiplicative set generated by $j$ ! and set

$$
\left(\begin{array}{c}
w \\
j
\end{array}\right):=\frac{\prod_{k=0}^{j-1}(w-k)}{j !} \in S^{-1} A .
$$

In particular $\left(\begin{array}{c}w \\ 0\end{array}\right)=1$ for any $w$, and $\left(\begin{array}{c}w \\ j\end{array}\right)=0$ for $w=0, \ldots, j-1$.

Let us first recall:

Theorem 1.2 (Macaulay's Theorem). A function $H: \mathbb{N} \rightarrow \mathbb{N}$ is the Hilbert function of a standard graded algebra if and only if $H(0)=1$ and $H(\mu+1) \leq H(\mu)^{\langle\mu\rangle}$ for $\mu \geq 1$. Moreover, $H(\mu+1)=H(\mu)^{\langle\mu\rangle}$ for $\mu \gg 0$.

Proposition 1.3. Let $P$ be an integral valued polynomial with positive leading coefficient. The following are equivalent:

(i) $P$ is the Hilbert polynomial of a projective scheme of dimension $d$.

(ii) There exists $b_{d}, \ldots, b_{0} \in \mathbb{N}$ such that

$$
P(\mu)=\left[b_{d}, \ldots, b_{0}\right]_{\mu} \text { for } \mu \gg 0 .
$$

Proof. (i) $\Rightarrow$ (ii) is a direct corollary of Macaulay's Theorem. For the opposite implication, set $b:=b_{0}+\cdots+b_{d}$ and choose $n$ such that $\left(\begin{array}{c}b+n \\ n\end{array}\right) \geq P(b)$; by Macaulay's Theorem the function

$$
\mu \longmapsto \begin{cases}\left(\begin{array}{c}
\mu+n \\
n
\end{array}\right) & \text { if } \mu<b \\
P(\mu) & \text { if } \mu \geq b\end{cases}
$$

is a Hilbert function. 
Remark 1.4. If $e_{1} \geq e_{2} \geq \cdots \geq e_{b} \geq 0$ and $\mu \geq b$, then

$$
\left\langle e_{1}, \ldots, e_{b}\right\rangle_{\mu}=\left(\begin{array}{c}
e_{1}+\mu \\
e_{1}
\end{array}\right)+\left(\begin{array}{c}
e_{2}+\mu-1 \\
e_{2}
\end{array}\right)+\cdots+\left(\begin{array}{c}
e_{b}+\mu-b+1 \\
e_{b}
\end{array}\right)
$$

is a polynomial function of $\mu$ of degree $e_{1}$. So, in Proposition 1.3, (ii) may be replaced by

(ii)' $^{\prime}$ There exists $b_{d}, \ldots, b_{0} \in \mathbb{N}$ such that

$$
P(\mu)=\left[b_{d}, \ldots, b_{0}\right]_{\mu} \text { for } \mu \geq b_{0}+\cdots+b_{d} .
$$

We will call $\left[b_{d}, \ldots, b_{0}\right]$ the Macaulay representation of the Hilbert polynomial $P$. We will now give a characterization of

$$
\mathcal{H} \subset \operatorname{Int}(\mathbb{Z}) \subset \mathbb{Q}[t]
$$

the set of integral polynomials that satisfy the two equivalent conditions of Proposition 1.3 in terms of the representation $P=\left(a_{d}, \ldots, a_{0}\right)$. To that end we study the conversion between the representations $P=\left(a_{d}, \ldots, a_{0}\right)$ and $P=\left[b_{d}, \ldots, b_{0}\right]$.

Lemma 1.5. Let $P \in \mathcal{H}$. The representations $P=\left(a_{d}, \ldots, a_{0}\right)$ and $P=\left[b_{d}, \ldots, b_{0}\right]$ may be computed one from another as follows. Set $A_{i}:=a_{d}+\cdots+a_{i}$ and $B_{i}:=$ $b_{d}+\cdots+b_{i}$. Then for $0 \leq j \leq d$,

$$
A_{j}=\sum_{i=0}^{d-j}(-1)^{i}\left(\begin{array}{c}
B_{i+j} \\
i+1
\end{array}\right) .
$$

Proof. We proceed by recursion on $d$. If $d=0$, one has $A_{0}=a_{0}=b_{0}=B_{0}$. If $d>0$, notice that if $\Delta P(t):=P(t)-P(t-1)$, one has $\Delta P \in \mathcal{H}$ and $\Delta P=$ $\left(a_{d}, \ldots, a_{1}\right)=\left[b_{d}, \ldots, b_{1}\right]$ as

$$
\left(\begin{array}{c}
w \\
i
\end{array}\right)-\left(\begin{array}{c}
w-1 \\
i
\end{array}\right)=\left(\begin{array}{c}
w-1 \\
i-1
\end{array}\right)
$$

Therefore, by induction we already know that $A_{j}=\sum_{i=0}^{d-j}(-1)^{i}\left(\begin{array}{c}B_{i+j} \\ i+1\end{array}\right)$ for $j>0$. Now for $j=0$ we have $A_{0}=P(0)$, so that it remains to check that the value at 0 of the polynomial $\left[b_{d}, \ldots, b_{0}\right]$ in terms of the $B_{i}$ 's is $B_{0}-\left(\begin{array}{c}B_{1} \\ 2\end{array}\right)+\cdots+(-1)^{d}\left(\begin{array}{c}B_{d} \\ d+1\end{array}\right)$. We induct on $B_{0}$; if $B_{0}=1$, then $P=(1,0, \ldots, 0)=[1,0, \ldots, 0]$ and the formulas give $A_{i}=B_{i}$ as $\left(\begin{array}{c}1 \\ i+1\end{array}\right)=0$ for $i$ positive. We now assume that $B_{0} \geq 2$.

Let us set $B_{d+1}:=0$ and

$$
P_{i}(t):=\sum_{k=B_{i}-1}^{B_{i+1}}\left(\begin{array}{c}
t+i-k \\
i
\end{array}\right)
$$

for $0 \leq i \leq d$. By hypothesis, $P(\mu)=\sum_{i=0}^{d} P_{i}(\mu)$ for $\mu \in \mathbb{Z}$. Therefore $P(0)=$ $\sum_{i=0}^{d} P_{i}(0)$, and we get

$$
P_{i}(0)=(-1)^{i} \sum_{k=B_{i}-1}^{B_{i+1}}\left(\begin{array}{c}
k-1 \\
i
\end{array}\right)
$$

so that we have to prove the identity

$$
\sum_{i=0}^{d}(-1)^{i} \sum_{k=B_{i}-1}^{B_{i+1}}\left(\begin{array}{c}
k-1 \\
i
\end{array}\right)=\sum_{i=0}^{d}(-1)^{i}\left(\begin{array}{c}
B_{i} \\
i+1
\end{array}\right) .
$$


Let $\ell:=\min \left\{i \mid P_{i} \neq 0\right\}$, so that $B_{0}=B_{1}=\cdots=B_{i}>B_{i+1}$. We have $P=\left[b_{n}, \ldots, b_{\ell}, 0, \ldots, 0\right] ;$ let us set $Q:=\left[b_{n}, \ldots, b_{\ell+1}, b_{\ell}-1,0, \ldots, 0\right]$. By induction we know that (1.1) is satisfied for $Q$. Therefore setting $B_{i}^{\prime}:=B_{i}-1$ for $i \leq \ell$ and $B_{i}^{\prime}:=B_{i}$ for $i>\ell$, we have (1.1), with the $B_{i}^{\prime}$ 's in place of the $B_{i}$ 's.

Taking the difference, we have to prove that

$$
(-1)^{\ell}\left(\begin{array}{c}
B_{\ell}-2 \\
\ell
\end{array}\right)=\sum_{i=0}^{\ell}(-1)^{i}\left[\left(\begin{array}{c}
B_{\ell} \\
i+1
\end{array}\right)-\left(\begin{array}{c}
B_{\ell}-1 \\
i+1
\end{array}\right)\right] .
$$

As $\left(\begin{array}{c}B_{\ell} \\ i+1\end{array}\right)-\left(\begin{array}{c}B_{\ell}-1 \\ i+1\end{array}\right)=\left(\begin{array}{c}B_{\ell}-1 \\ i\end{array}\right)$, this follows from the identity

$$
\sum_{i=0}^{\ell}(-1)^{i}\left(\begin{array}{c}
w \\
i
\end{array}\right)=(-1)^{\ell}\left(\begin{array}{c}
w-1 \\
\ell
\end{array}\right)
$$

which is easy to prove by induction on $\ell$.

Example 1.6. If $P$ is respectively $(a),(a, b)$ or $(a, b, c)$ with $a \neq 0$, the values of $B_{0}$ are respectively $a,\left(\begin{array}{c}a+1 \\ 2\end{array}\right)+b$ and $c+b+a+\left(\begin{array}{c}b+\left(\begin{array}{c}a+1 \\ 2\end{array}\right) \\ 2\end{array}\right)-\left(\begin{array}{c}a \\ 3\end{array}\right)$.

The respective characterizations of being the Hilbert polynomial of a scheme of dimension 0,1 and 2 are respectively:

- $a>0$,

- $a>0$ and $b \geq-\left(\begin{array}{l}a \\ 2\end{array}\right)$,

- $a>0, b \geq-\left(\begin{array}{l}a \\ 2\end{array}\right)$ and $c \geq-\left(\begin{array}{c}b+\left(\begin{array}{c}a+1 \\ 2\end{array}\right) \\ 2\end{array}\right)+\left(\begin{array}{c}a+1 \\ 3\end{array}\right)$.

In the case of dimension 1, with the more familiar $P(t)=d t+1-g$ the inequalities become $d \geq 1$ and $g \leq \frac{(d-1)(d-2)}{2}$.

\section{Regularity bounds for schemes with a given Hilbert polynomial}

Notation 2.1. If $I$ is a homogeneous ideal in a polynomial ring $R=k\left[X_{0}, \ldots, X_{n}\right]$, let $\mathfrak{m}:=\left(X_{0}, \ldots, X_{n}\right)$ be the graded maximal ideal of $R$, and denote respectively by $H$ and $P$ the Hilbert function and polynomial of $R / I$. We define:

- $\sigma(H):=\min \{\mu \mid H(\nu)=P(\nu), \forall \nu>\mu\}$,

- lex $(H)$ is the lex-segment ideal in $R$ corresponding to the function $H$,

- $B(P):=b_{0}+\cdots+b_{d}$ if $P=\left[b_{d}, \ldots, b_{0}\right]$.

If $M$ is a finitely generated graded $R$-module, we define:

- $\beta_{i, j}(M):=\operatorname{dim}_{k} \operatorname{Tor}_{i}^{R}(M, k)_{j}, b_{i}(M):=\max \left\{j \mid \beta_{i, j}(M) \neq 0\right\}$,

- $a_{i}(M):=\max \left\{j \mid H_{\mathfrak{m}}^{i}(M)_{j} \neq 0\right\}$,

- $\operatorname{reg}(M):=\max \left\{b_{i}(M)-i\right\}=\max \left\{a_{i}(M)+i\right\}$,

- $r(M):=\max \left\{a_{i}(M)\right\}=\max \left\{b_{i}(M)\right\}-n-1$.

(We used the convention $\max \emptyset=-\infty$.)

We first recall some classical facts on the invariants above.

Reminder 2.2. With the notations above,

(i) For all $i$ and all $j, \beta_{i, j}(I) \leq \beta_{i, j}(\operatorname{lex}(H))$, in particular $\operatorname{reg}(I) \leq \operatorname{reg}(\operatorname{lex}(H))$.

(ii) For any $\mu \in \mathbb{Z}$,

$$
H(\mu)=P(\mu)+\sum_{i=0}^{d}(-1)^{i} \operatorname{dim}_{k} H_{\mathfrak{m}}^{i}(R / I)_{\mu} .
$$


In particular $\sigma(H) \leq r(I)=r(R / I) \leq \operatorname{reg}(R / I)=\operatorname{reg}(I)-1$. Moreover $\sigma(H) \neq \operatorname{reg}(R / I)$ if and only if one of the two following equivalent conditions is satisfied:

(1) $\operatorname{reg}(R / I) \neq a_{0}(R / I)$,

(2) $\operatorname{reg}(I) \neq b_{n}(I)-n$.

(iii) The following are equivalent:

(1) $\operatorname{lex}(H)$ is generated in degrees $\leq r$,

(2) $\operatorname{reg}(\operatorname{lex}(H)) \leq r$,

(3) $H(\mu+1)=H(\mu)^{\langle\mu\rangle}$ for $\mu \geq r$.

In particular,

$$
\operatorname{reg}(\operatorname{lex}(H))=\min \left\{\mu>\sigma(H) \mid \forall \nu \geq \mu, P(\nu)^{\langle\nu\rangle}=P(\nu+1)\right\} .
$$

Proof. (i) was first proved in characteristic 0 in $\mathrm{Bi}$ ] and $\mathrm{Hu}$, and in the general case in [Pa] (see also [CGP for a short argument in characteristic 0). Fact (ii) may for example be found in $[\mathrm{BH}, 4.3 .5$, and the equivalence of the strict inequality with condition (1) is evident. Now $a_{0}(R / I)=b_{n}(I)-n-1$ by lemma 2.3 below, which gives the equivalence with (2).

For (iii), (1) is equivalent to (2) due to the Eliahou-Kervaire resolution of lex $(H)$. The equivalence of (1) and (3) is a lemma of Macaulay ( $[\mathrm{Gr}, 3.7$ or $[\mathrm{BH}], 4.2 .9)$.

Lemma 2.3. Let $I \subset R$ be a homogeneous ideal. If $H_{\mathfrak{m}}^{0}(R / I)_{\mu+1}=0$, then

$$
H_{\mathfrak{m}}^{0}(R / I)_{\mu} \simeq \operatorname{Tor}_{n}^{R}(I, k)_{\mu+1+n}=\operatorname{Tor}_{n+1}^{R}(R / I, k)_{\mu+n+1} .
$$

In particular, $a_{0}(R / I)=b_{n}(I)-n-1$.

Proof. The second equality is evident. One has

$$
\operatorname{Tor}_{n+1}^{R}(R / I, k) \simeq \operatorname{Socle}\left(H_{\mathfrak{m}}^{0}(R / I)\right)[-n-1] .
$$

If $H_{\mathfrak{m}}^{0}(R / I)_{\mu+1}=0$, then $\mathfrak{m} H_{\mathfrak{m}}^{0}(R / I)_{\mu}=0$ so that $H_{\mathfrak{m}}^{0}(R / I)_{\mu}=\operatorname{Socle}\left(H_{\mathfrak{m}}^{0}(R / I)\right)_{\mu}$.

Remark 2.4. More generally one can check that

$$
\max _{0 \leq i \leq k}\left\{a_{i}(R / I)+i\right\}=\max _{n-k \leq i \leq n}\left\{b_{i}(I)-i-1\right\} .
$$

Theorem 2.5. Let $I \subset R$ be a homogeneous ideal. With the notations above,

(i) $\operatorname{reg}(\operatorname{lex}(H))=\max \{B(P), \sigma(H)+1\}=\max \{B(P), r(R / I)+1\}$.

(ii) If $\operatorname{reg}(I) \neq \operatorname{reg}(\operatorname{lex}(H))$ or $\operatorname{reg}(R / I) \neq a_{0}(R / I)$, then $\operatorname{reg}(\operatorname{lex}(H))=B(P)$.

Proof. By Reminder 2.2 (i) and (ii), $\sigma(H)+1 \leq r(R / I)+1 \leq \operatorname{reg}(I) \leq \operatorname{reg}(\operatorname{lex}(H))$, and the second inequality is strict if $\operatorname{reg}(R / I) \neq a_{0}(R / I)$. This shows that the second statement is a consequence of the first one, and that it suffices to prove that $\operatorname{reg}(\operatorname{lex}(H))=\max \{B(P), \sigma(H)+1\}$, which follows from Reminder 2.2 (iii) and Remark 1.4

For a function $F: \mathbb{Z} \rightarrow \mathbb{Z}$, we set $\Delta F(m):=F(m)-F(m-1)$.

Corollary 2.6. If $X$ is a projective scheme with Hilbert polynomial $P, I_{X}$ the saturated ideal defining $X$ and $H_{X}$ the Hilbert function of $R / I_{X}$, then

$$
\operatorname{reg}\left(I_{X}\right) \leq \operatorname{reg}\left(\operatorname{lex}\left(\Delta H_{X}\right)\right)=\max \left\{\sigma\left(\Delta H_{X}\right)+1, B(\Delta P)\right\} \leq B(P) .
$$


Proof. By definition $I_{X}$ is the biggest homogeneous ideal defining $X$ and is therefore saturated, so one may choose a linear form $\ell$ which is not a zero divisor in $R / I_{X}$. The ideal $I_{X}+(\ell)$ has the same regularity as the one of $I_{X}$ and its Hilbert function is $\Delta H_{X}$ so that Reminder 2.2 (i) gives the first inequality. One also notices that $B(\Delta P) \leq B(P)$ which shows the last inequality because $\sigma\left(\Delta H_{X}\right) \leq \sigma\left(H_{X}\right)+1$ and $B(P)=\operatorname{reg}\left(\operatorname{lex}\left(H_{X}\right)\right) \geq \sigma\left(H_{X}\right)+2$ by Reminder 2.2 (ii).

It may a priori seem easier to bound $\sigma\left(H_{X}\right)$ than to bound $\operatorname{reg}\left(I_{X}\right)$. The following remark shows that it is equivalent in many cases.

Remark 2.7. Let $\mathcal{P}$ be a property of embedded projective schemes and $N(X)$ a numerical invariant attached to such a scheme $X$. Assume that if $X \subseteq \mathbb{P}_{n}$ satisfies $\mathcal{P}$ and $H$ is a general hyperplane, then $X \cap H \subseteq H \simeq \mathbb{P}_{n-1}$ satisfies $\mathcal{P}$ and $N(X \cap H) \leq$ $N(X)$. We denote by $I_{X} \subseteq R$ the defining ideal of $X$ and by $H_{X}$ the Hilbert function of $R / I_{X}$. Then the following are equivalent:

(i) If $X$ satisfies $\mathcal{P}, \operatorname{reg}\left(R / I_{X}\right) \leq N(X)$.

(ii) If $X$ satisfies $\mathcal{P}, \sigma\left(H_{X}\right) \leq N(X)-1$.

(iii) If $X$ satisfies $\mathcal{P},\left(R / I_{X}\right)_{\mu}=H^{0}\left(X, \mathcal{O}_{X}(\mu)\right)$ for $\mu \geq N(X)$.

Note that (iii) is equivalent to $a_{1}\left(R / I_{X}\right) \leq N(X)-1$ so that it is clear that (i) implies both (ii) and (iii). We prove the other implications by recursion on the dimension of $X$ (the zero dimensional case is clear). The key point is that if $H$ is a general hyperplane,

$$
\operatorname{reg}\left(R / I_{X}\right)=\max \left\{a_{1}\left(R / I_{X}\right)+1, \operatorname{reg}\left(R / I_{X \cap H}\right)\right\},
$$

which is a direct consequence of the long exact sequence

$$
\begin{aligned}
H_{\mathfrak{m}}^{1}\left(R / I_{X}\right) \rightarrow H_{\mathfrak{m}}^{1}\left(R / I_{X \cap H}\right) \rightarrow H_{\mathfrak{m}}^{2}\left(R / I_{X}\right)[-1] \\
\quad \rightarrow H_{\mathfrak{m}}^{2}\left(R / I_{X}\right) \rightarrow H_{\mathfrak{m}}^{2}\left(R / I_{X \cap H}\right) \rightarrow \cdots .
\end{aligned}
$$

This shows that (iii) implies (i). For (ii) $\Rightarrow(\mathrm{i})$, notice that if $\sigma\left(H_{X}\right)<\operatorname{reg}\left(R / I_{X}\right)-1$, then $\operatorname{reg}\left(R / I_{X}\right) \neq a_{1}\left(R / I_{X}\right)+1$ by Reminder 2.2 (ii) and therefore $\operatorname{reg}\left(R / I_{X}\right)=$ $\operatorname{reg}\left(R / I_{X \cap H}\right)$ which proves the claim by induction.

For example one may choose for property $\mathcal{P}: X$ satisfies $S_{k}, X$ is smooth in codimension $l, X$ is irreducible, $X$ is equidimensional, or any conjunction of some of these properties. For $N(X)$ one may choose the degree of $X$, or the degree of $X$ minus the embedding codimension of $X$ if $X$ is irreducible and reduced, or the minimum over the sets of equations defining $X$ of the maximal degree of these equations.

\section{Bounds For the Castelnuovo-Mumford Regularity OF THE LEX-SEGMENT IDEAL ASSOCIATED TO A GRADED COMPLETE INTERSECTION, AND APPLICATIONS}

Let $R:=k\left[X_{0}, \ldots, X_{n}\right]$. We will now estimate the value of the regularity of the lex-segment ideal associated to the Hilbert function of a graded complete intersection (which is equal to the maximal degree of a minimal generator of this lex-segment ideal), because the actual closed formulas that one may derive from Lemma1.5 are hard to deal with.

Lemma 3.1. Let $H$ be the Hilbert function of a complete intersection given by forms of degrees $d_{1}, \ldots, d_{r}$ in $R$. Assume for simplicity that $d_{i} \geq 2$ for all $i$; then 
- if $r=n+1, \operatorname{reg}(\operatorname{lex}(H))=d_{1}+\cdots+d_{r}-n$,

- if $r=n, \operatorname{reg}(\operatorname{lex}(H))=d_{1} \cdots d_{r}$,

- if $r \leq n, \operatorname{reg}(\operatorname{lex}(H)) \leq\left[d_{1} \cdots d_{r}\right]^{2^{n-r}}$.

The proof will be given later. It is possible to improve the bound for $r<n$; for example we checked that if $n-r$ is at most 3 , then $\operatorname{reg}(\operatorname{lex}(H)) \leq 2\left[\frac{d_{1} \cdots d_{r}}{2}\right]^{2^{n-r}}$, which is quite optimal and seems to hold in general.

Lemma 3.2. Let $P=\left(a_{D}, \ldots, a_{0}\right)$ be the Hilbert polynomial of a complete intersection of degrees $\left(d_{1}, \ldots, d_{r}\right)$. Then, for $0 \leq j \leq D$,

$$
a_{D-j}=(-1)^{j} \sum_{i_{1}=0}^{d_{1}-1} \cdots \sum_{i_{r}=0}^{d_{r}-1}\left(\begin{array}{c}
i_{1}+\cdots+i_{r} \\
j
\end{array}\right)
$$

and

$$
\left|a_{D-j}\right| \leq \frac{d_{1} \cdots d_{r}}{j+1}\left(\begin{array}{c}
d_{1}+\cdots+d_{r}-r \\
j
\end{array}\right) .
$$

The second statement follows from the first and the following upper bound.

Lemma 3.3. Let $m_{1}, \ldots, m_{r}$ and $j$ be non-negative integers. Then

$$
\sum_{i_{1}=0}^{m_{1}} \cdots \sum_{i_{r}=0}^{m_{r}}\left(\begin{array}{c}
i_{1}+\cdots+i_{r} \\
j
\end{array}\right) \leq \frac{\left(m_{1}+1\right) \cdots\left(m_{r}+1\right)}{j+1}\left(\begin{array}{c}
m_{1}+\cdots+m_{r} \\
j
\end{array}\right) .
$$

Proof of 3.3 (Suggested by Joseph Oesterlé). Set $m:=\left(m_{1}, \ldots, m_{r}\right)$ and denote $F(m):=\sum_{i_{1}=0}^{m_{1}} \cdots \sum_{i_{r}=0}^{m_{r}}\left({ }^{i_{1}+\cdots+i_{r}}\right)$ and $G(m):=\frac{\left(m_{1}+1\right) \cdots\left(m_{r}+1\right)}{j+1}\left({ }^{m_{1}+\cdots+m_{r}}\right)$.

We have to prove the term-by-term inequality of series $S F_{j}(T) \leq S G_{j}(T)$, with $S F_{j}(T):=\sum_{m} F_{j}(m) T^{m}$ and $S G_{j}(T):=\sum_{m} G_{j}(m) T^{m}$. As $\left(1-T_{k}\right)^{-1}$ is a series with non-negative coefficients, it suffices to show that

$$
\left(1-T_{1}\right) \cdots\left(1-T_{r}\right) S F_{j}(T) \leq\left(1-T_{1}\right) \cdots\left(1-T_{r}\right) S G_{j}(m) .
$$

First notice that

$$
\left(1-T_{1}\right) \cdots\left(1-T_{r}\right) S F_{j}(T)=\sum_{m}\left(\begin{array}{c}
m_{1}+\cdots+m_{r} \\
j
\end{array}\right) T^{m} .
$$

Let $D G_{j}^{k}(m)$ be the coefficient of $T^{m}$ in $(j+1)\left(1-T_{1}\right) \cdots\left(1-T_{k}\right) S G_{j}(T)$, so that we have to show $D G_{j}^{r}(m) \geq(j+1)\left({ }^{m_{1}+\cdots+m_{r}}\right)$. A recursion on $k$ from 0 to $r$ easily shows that

$$
D G_{j}^{k}(m)=\prod_{\ell>k}\left(m_{\ell}+1\right) \sum_{S \subset[k]} \prod_{s \in S} m_{s}\left(\begin{array}{c}
m_{1}+\cdots+m_{r}-|S| \\
j-|S|
\end{array}\right) .
$$

We now bound $D G_{j}^{r}(m)$ by the sum of terms for which $|S|$ is equal to 0 or 1 ,

$$
\begin{aligned}
D G_{j}^{r}(m) & \geq\left(\begin{array}{c}
m_{1}+\cdots+m_{r} \\
j
\end{array}\right)+\left(m_{1}+\cdots+m_{r}\right)\left(\begin{array}{c}
m_{1}+\cdots+m_{r}-1 \\
j-1
\end{array}\right) \\
& =(j+1)\left(\begin{array}{c}
m_{1}+\cdots+m_{r} \\
j
\end{array}\right) .
\end{aligned}
$$

This proves the inequality. 
Proof of Lemma 3.2. The identities $1-T_{k}^{d_{k}}=\left(1-T_{k}\right) \sum_{i=0}^{d_{k}-1} T_{k}^{i}$ and the fact that $P(\mu)=\left(\begin{array}{c}\mu+D \\ D\end{array}\right)$ if $d_{k}=1$ for all $k$ show that

$$
P(\mu)=\sum_{i_{1}=0}^{d_{1}-1} \cdots \sum_{i_{r}=0}^{d_{r}-1}\left(\begin{array}{c}
\mu+D-\left(i_{1}+\cdots+i_{r}\right) \\
D
\end{array}\right) .
$$

Therefore, the identity $\left(\begin{array}{c}\mu+D-i \\ D\end{array}\right)=\sum_{j=0}^{D}(-1)^{j}\left(\begin{array}{c}\mu+D-j \\ D-j\end{array}\right)\left(\begin{array}{c}i \\ j\end{array}\right)$ provides the first claim and the bound follows from Lemma 3.3.

Proof of Lemma 3.1. If we set $\pi:=d_{1} \cdots d_{r}$ and $\sigma:=d_{1}+\cdots+d_{r}-r$, we know from Lemma 3.2 that $\left|a_{D-j}\right| \leq \frac{\pi}{j+1}\left(\begin{array}{c}\sigma \\ j\end{array}\right)$.

We now use Lemma 1.5 and an induction on $i$ to show that $B_{D-i} \leq \pi^{2^{i}}$. Note that $B_{D}=\pi, B_{D-1}=\left(\begin{array}{c}\pi+1 \\ 2\end{array}\right)-\frac{1}{2} \pi \sigma \leq \pi^{2}$ and that we may assume that $r \geq 2$ so that $\pi \geq \sigma$. Let $\ell$ be the integral part of $\frac{i}{2}$ and set $B_{D+1}:=0$. By Lemma 1.5, for $i \geq 2$, the induction gives

$$
\begin{aligned}
B_{D-i} & =a_{D-i}+\cdots+a_{D}+\left(\begin{array}{c}
B_{D-i+1} \\
2
\end{array}\right)-\left(\begin{array}{c}
B_{D-i+2} \\
3
\end{array}\right)+\cdots+(-1)^{i+1}\left(\begin{array}{c}
B_{D} \\
i+1
\end{array}\right) \\
& \leq \sum_{j=0}^{\ell} a_{D-2 j}+\sum_{j=0}^{\ell}\left(\begin{array}{c}
B_{D-i+2 j+1} \\
2 j+2
\end{array}\right) \\
& \leq \sum_{j=0}^{\ell} \frac{\pi}{2 j+1}\left(\begin{array}{c}
\sigma \\
2 j
\end{array}\right)+\sum_{j=0}^{\ell}\left(\begin{array}{c}
\pi^{2^{i-2 j-1}} \\
2 j+2
\end{array}\right) \quad \text { (by Lemma } 3.2 \text { and induction) } \\
& \left.\leq \sum_{j=0}^{\ell} \frac{\pi^{2 j+1}}{(2 j+1) !}+\sum_{j=0}^{\ell} \frac{\pi^{(2 j+2) \times 2^{i-2 j-1}}}{(2 j+2) !} \quad \text { (because } \sigma \leq \pi\right) \\
& \leq \sum_{j=0}^{\ell} \pi^{2 j+1} 2^{2 j}+\frac{1}{2} \pi^{2^{i}}+\frac{1}{24} \sum_{j=1}^{\ell} \pi^{2^{i-j}}\left(\text { notice that } 2^{t} \geq 2 t \text { for } t \geq 2\right) \\
& \leq 4\left(\frac{\pi}{2}\right)^{2 \ell+1}+\frac{1}{2} \pi^{2^{i}}+\frac{1}{12} \pi^{2^{i-1}} \\
& \leq \pi^{2^{i}}\left(\frac{1}{2 \pi}+\frac{1}{2}+\frac{1}{12 \pi^{2}}\right) \quad\left(\text { as } 2^{i} \geq \max \left\{2^{i-1}+2,2 \ell+2\right\}\right) .
\end{aligned}
$$

Corollary 3.4. Let $S=R / I$ be a standard graded algebra whose Hilbert function $H$ is the one of a complete intersection of degrees $d_{1}, \ldots, d_{r}$ with $r \leq n$. Then

$$
\operatorname{reg}(I) \leq\left[d_{1} \cdots d_{r}\right]^{2^{n-r}} .
$$

Proof. By Reminder $2.2(\mathrm{i}), \operatorname{reg}(I) \leq \operatorname{reg}(\operatorname{lex}(H))$ and the estimate of Lemma 3.1 gives the bound.

Lemma 3.5. Let $f_{1}, \ldots, f_{s}$ be forms of degrees $d_{1}, \ldots, d_{s}$ in $R, I$ the ideal they generate and $r:=\operatorname{codim}(I)$.

(i) If $r=s, \operatorname{reg}(I)=d_{1}+\cdots+d_{s}-s+1$. Also if $J$ is an intersection of prime ideals associated to $R / I$, there exists $h \in R$ such that $\operatorname{deg} h \leq d_{1}+\cdots+d_{s}-s$ and $J=I:(h)$. One has $\operatorname{reg}(J) \leq \max \{\operatorname{reg}(I), \operatorname{reg}(I+(h))-\operatorname{deg} h+1\}$ and $\operatorname{reg}(J)=\operatorname{reg}(I+(h))-\operatorname{deg} h+1$ if $\operatorname{reg}(J)>\operatorname{reg}(I)$. 
(ii) Let $\omega$ be an admissible monomial order on $R$. Then there exists a complete intersection $I^{\prime}$ of degrees $d_{1}, \ldots, d_{s}$ in $R^{\prime}:=R\left[Y_{1}, \ldots, Y_{s-r}\right]$ and an order $\omega^{\prime}$ refining $\omega$ such that $\operatorname{in}_{\omega}(I)=\operatorname{in}_{\omega^{\prime}}\left(I^{\prime}\right) \bmod \left(Y_{1}, \ldots, Y_{s-r}\right)$. In particular

$$
b_{0}\left(\operatorname{in}_{\omega}(I)\right) \leq b_{0}\left(\operatorname{in}_{\omega^{\prime}}\left(I^{\prime}\right)\right) .
$$

Proof. Part (i) easily follows from Ch, Th. 27, Th. 28 and Lem. 31. With the notations of [Ch], Th. 28, if $J=\mathfrak{P}_{i_{1}} \cap \cdots \cap \mathfrak{P}_{i_{k}}$, choose $h:=t_{i_{1}}+\cdots+t_{i_{k}}$ so that $J=I:(h)$ and then (i) follows with the same arguments as in the proof of Ch], Lem. 31, replacing $t_{i}$ by $h$.

For (ii) we may assume that $d_{1} \geq d_{2} \geq \cdots \geq d_{s}$ and that the first $r$ forms $f_{1}, \ldots, f_{r}$ form a graded complete intersection. Now the ideal $I^{\prime}:=\left(f_{1}, \ldots, f_{r}, f_{r+1}\right.$ $\left.-Y_{1}^{d_{r+1}}, \ldots, f_{s}-Y_{s-r}^{d_{s}}\right)$ is a graded complete intersection in $R^{\prime}$, and the minimal generators of $\operatorname{in}_{\omega}(I)$ are part of the ones of $\operatorname{in}_{\omega^{\prime}}\left(I^{\prime}\right)$ because $\operatorname{in}_{\omega}(I)=\operatorname{in}_{\omega^{\prime}}\left(I^{\prime}\right) \bmod$ $\left(Y_{1}, \ldots, Y_{s-r}\right)$ if we choose $\omega^{\prime}$ such that it refines the partial rev-lex order on the blocks $X, Y$.

In particular (ii) gives the following.

Corollary 3.6. Let $I \subset R$ be an ideal generated by polynomials of degrees $d_{1}, \ldots, d_{s}$ and let $r$ be the codimension of $I$. Then for any admissible monomial order and any coordinates,

$$
b_{0}(\operatorname{in}(I)) \leq\left[d_{1} \cdots d_{s}\right]^{2^{n-r}} .
$$

Corollary 3.7. Let $J \subset R$ be an intersection of prime ideals associated to a complete intersection $I$ of degrees $d_{1}, \ldots, d_{s}$. Then for any admissible monomial order and any coordinates,

$$
b_{0}(\operatorname{in}(J)) \leq\left[d_{1} \cdots d_{s}\left(d_{1}+\cdots+d_{s}-s\right)\right]^{2^{n-r}},
$$

unless $d_{i}=1$ for all $i$.

Proof. Let $\sigma:=d_{1}+\cdots+d_{s}-s$ and $h$ be as in Lemma 3.5(i). Consider $R^{\prime}:=R[Y]$ and $I^{\prime}:=I+\left(h-Y^{\sigma}\right)$.

Let $\omega^{\prime}$ be the order refining the admissible order $\omega$ of $R$, defined by : $X^{a} Y^{u}<{ }_{\omega^{\prime}}$ $X^{b} Y^{v} \Longleftrightarrow u>v$ or $u=v$ and $a<_{\omega} b$. Then, $\operatorname{in}(I:(h))=\operatorname{in}\left(I^{\prime}:(h)\right) \cap$ $R=\left(\operatorname{in}\left(I^{\prime}\right):\left(Y^{\sigma}\right)\right) \cap R$. Therefore, the minimal generators of in $(I:(h))$ are the specialization at $Y=1$ of minimal generators of in $\left(I^{\prime}\right)$ of degree in $Y$ at most $\sigma$.

Remark 3.8. (i) For any monomial ideal $J$ in $R, \operatorname{reg}(J) \leq(n+1) b_{0}(J)$, as follows from the Taylor resolution. Note also that if $I$ is an ideal and $\omega$ an admissible monomial order, $\operatorname{reg}(I) \leq \operatorname{reg}\left(\operatorname{in}_{\omega}(I)\right)$ and if $I$ is saturated,

$$
b_{0}(I) \leq \operatorname{reg}(I) \leq n b_{0}\left(\operatorname{in}_{\omega}(I)\right) .
$$

(ii) If $J$ is a stable monomial ideal in $R$ (i.e., $X_{i} m \in J \Rightarrow X_{j} m \in J, \forall j \leq i$ ), setting $\max (m):=\max \left\{i \mid X_{i}\right.$ divides $\left.m\right\}$ and denoting by $G(J)$ the set of minimal monomial generators of $J$, the Eliahou-Kervaire resolution shows that

$$
\beta_{i, i+j}(J)=\sum_{m \in G(J)_{j}}\left(\begin{array}{c}
\max (m) \\
i
\end{array}\right)
$$

and the non-zero terms in the sum are such that $\max (m) \geq i$. In particular,

$$
b_{k}(J)-k=\max \left\{j \mid \exists m \in G(J)_{j}, \max (m) \geq k\right\}
$$


so that $\operatorname{reg}(J)=b_{0}(J)$. For any admissible order, any initial ideal in generic coordinates is stable if the characteristic of $k$ is zero (Galligo's theorem).

\section{REFERENCES}

[Bi] A. Bigatti, Upper bounds for the Betti numbers of a given Hilbert function, Comm. in Algebra 21 (1993), 2317-2334. MR 94c:13014

[BH] W. Bruns and J. Herzog, Cohen-Macaulay Rings, Cambridge Stud. in Adv. Math. 39, Cambridge Univ. Press (1993). MR 95h:13020

[Ch] M. Chardin, Applications of some properties of the canonical module in computational projective algebraic geometry, J. Symbolic Computation 29 (2000), 527-544. MR 2001g:14091

[CGP] M. Chardin, V. Gasharov and I. Peeva, Maximal Betti numbers, Proc. Amer. Math. Soc. 130 (2002), 1877-1880.

[Go] G. Gotzmann, Eine Bedingung für die Flachheit und das Hilbertpolynom eines graduierten Ringes, Math. Z. 158 (1978), 61-70. MR 58:641

[Gr] M. Green, Generic initial ideals, in Six lectures on commutative algebra, Birkhäuser, Progress in Mathematics 166, (1998), 119-185. MR 99m:13040

[Hu] H. Hulett, Maximum Betti numbers of homogeneous ideals with a given Hilbert function, Comm. in Algebra 21 (1993), 2335-2350. MR 94c:13015

$[\mathrm{Mu}] \quad$ D. Mumford, Lectures on curves on an algebraic surface, Ann. of Math. Stud. 59, Princeton University Press (1966). MR 35:187

[Pa] K. Pardue, Deformation classes of graded modules and maximal Betti numbers, Illinois J. Math. 40 (1996), 564-585. MR 97g:13029

Institut de Mathématiques, CNRS \& Université Paris 6, 4, Place Jussieu, F-75252 PARIS CEDEX 05, France

E-mail address: chardin@math.jussieu.fr

LAMA, Université de Versailles \& CNRS, 45, avenue des États-Unis, F-78035 VerSAILLES CEDEX, FRANCE

E-mail address: moreno@math.uvsq.fr 